EGG-CODP-10473

DE93 005273

\title{
Motif, the Basics An Overview of the Widget Set
}

Fred R. McClurg

Published October 1992

\section{Idaho National Engineering Laboratory \\ EG\&G Idaho, Inc. Idaho Falls, Idaho 83415}

Prepared for the

U.S. Depariment of Energy

Under DOE Idaho Field Office

Contract DE-AC07-76ID015\%0 


\begin{abstract}
The Motif library provides programmers with a rich set of tools for building a graphical user interface with a three-dimensional appearance and a consistent method of interaction for controlling an Unix application. This Xt-based, high-level library presents an "object-oriented" approach to program design for programmers and allows end-users the flexibility to modify attributes of the interface.
\end{abstract}




\section{ACKNOWLEDGMENTS}

The author would like to express his appreciation to the Computational Services, Visualization and Imaging Team, EG\&G Idaho, Inc. 


\section{CONTENTS}

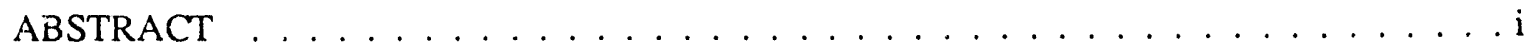

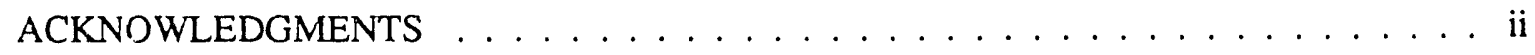

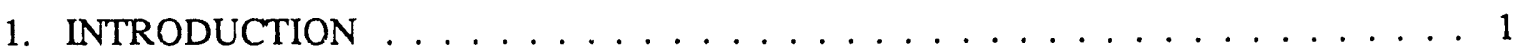

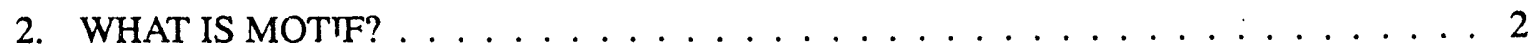

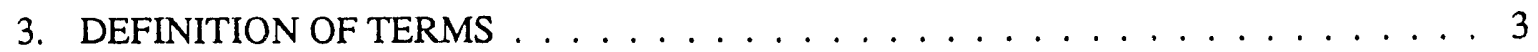

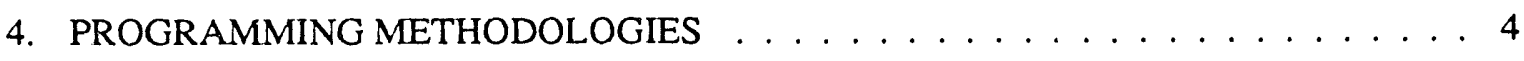

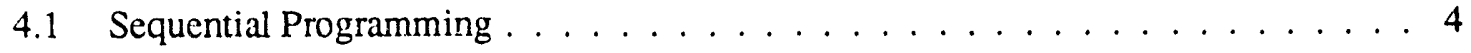

4.2 Event-driven Programming . . . . . . . . . . . . . . . . 4

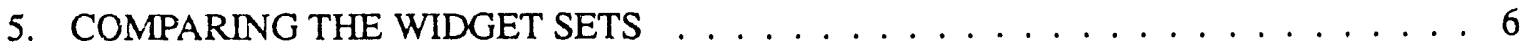

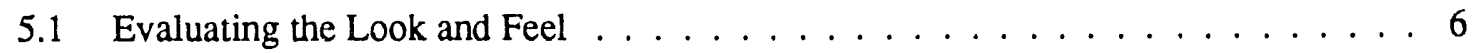

5.1 .1 "The Look" Considerations . . . . . . . . . . . . . . . . . . . 6

5.1 .2 The "Feel" Considerations . . . . . . . . . . . . . . 6

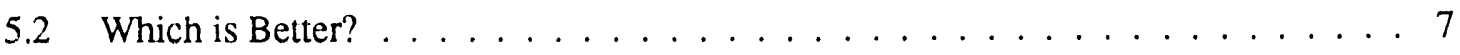

5.3 Is it Easy to Write Motif Code? . . . . . . . . . . . . . 7

6. WHAT ARE THE ADVANTAGES OF MOTIF? . . . . . . . . . . . . . . 9

7. WHAT ARE THE DISADVANTAGES OF MOTIF? . . . . . . . . . . . . 10

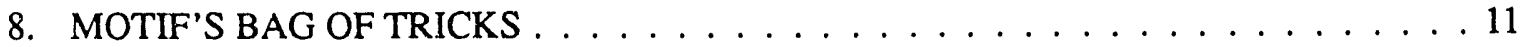

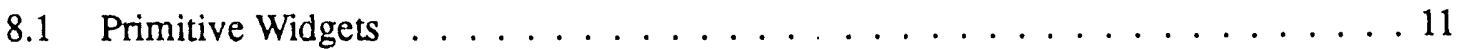

8.1 .1 Label . . . . . . . . . . . . . . . . . . . 11

8.1 .2 Push Button . . . . . . . . . . . . . . . . . 11

8.1 .3 Drawn Button . . . . . . . . . . . . . . . . . 12

8.1 .4 Arrow Button . . . . . . . . . . . . . . . . . 12

8.1 .5 Toggle Button . . . . . . . . . . . . . . . . . . . . . . . . . . .

8.1 .5 .1 Radio Boxes. . . . . . . . . . . . . . . . 12

8.1.5.2 Check Boxes. . . . . . . . . . . . . . . 13

8.16 Scale . . . . . . . . . . . . . . . . 13

8.1 .7 Scroll Bar . . . . . . . . . . . . . . . . . . . . . 14

8.1 .8 List Widget $\ldots \ldots \ldots \ldots \ldots \ldots \ldots$

8.1 .9 Text Widgets . . . . . . . . . . . . . . . . 14

8.1 .9 .1 Text Field. . . . . . . . . . . . . . . . 14

8.1 .9 .2 Scrolled Text. . . . . . . . . . . . . . . . . . . . . . . . . . . . . .

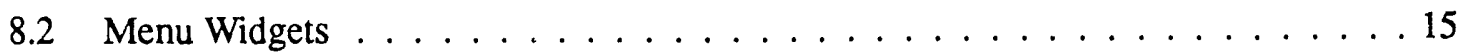

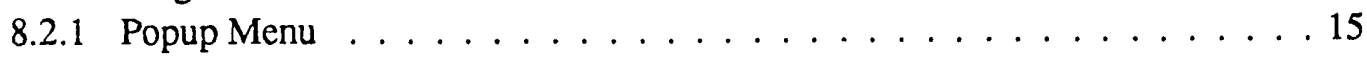

8.2 .2 Cascade Menu . . . . . . . . . . . . . . . . 16

8.2 .3 Option Menu . . . . . . . . . . . . . . . . 16

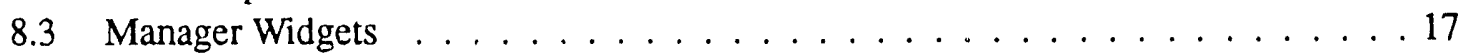

8.3 .1 Main Window . . . . . . . . . . . . . 17 
8.3 .2 Scrolled Window . . . . . . . . . . . . . . . . . . . 18

8.3 .3 Paned Window . . . . . . . . . . . . . . . . . 18

8.4 Dialog Widgets . . . . . . . . . . . . . . . . . . . . . . . . . . 19

8.4 .1 Selection Dialog . . . . . . . . . . . . . . . . . . . . . . . 19

8.4 .2 File Selection Dialog . . . . . . . . . . . . . . . . . . . . 19

8.4 .3 Command Dialog . . . . . . . . . . . . . . . . . . . . . . . . . . . . . . . . . . . . . . . . . . .

8.4 .4 Message Dialogs . . . . . . . . . . . . . . . . . . . 21

8.4.4.1 Error Dialog. . . . . . . . . . . . . . . . . . 21

8.4 .4 .2 Information Dialog. . . . . . . . . . . . . . 21

8.4 .4 .3 Question Dialog. . . . . . . . . . . . . . . . 21

8.4 .4 .4 Warning Dialog. . . . . . . . . . . . . . . 22

8.4 .4 .5 Working Dialog. . . . . . . . . . . . . . . 22

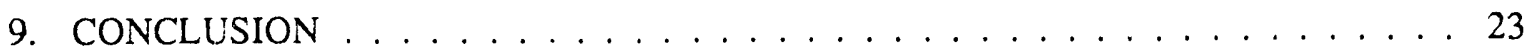

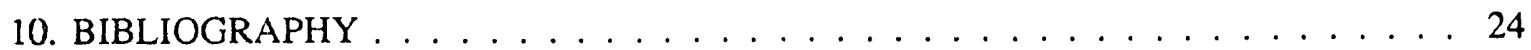




\section{FIGURES}

Figure 1. User Interface Model. . . . . . . . . . . . . . . . . . . . 2

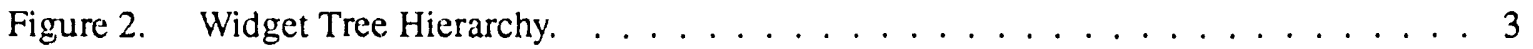

Figure 3. Standard Sequential Programming. . . . . . . . . . . . . . . . 4

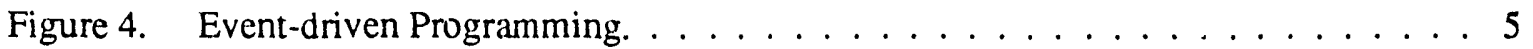

Figure 5. Simple Example Motif Application. . . . . . . . . . . . . . . 8

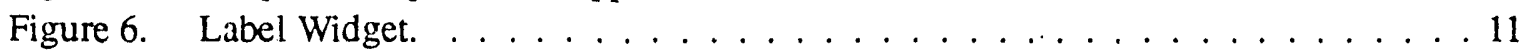

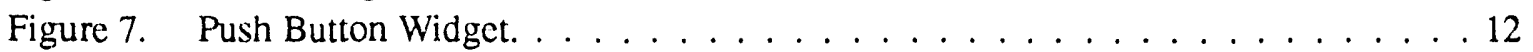

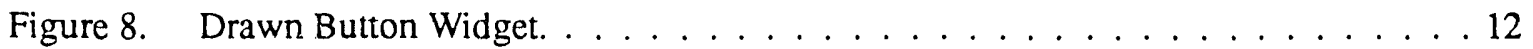

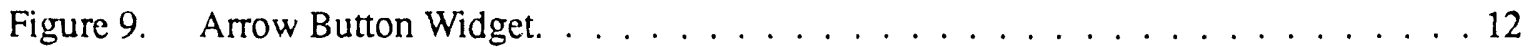

Figure 10. Toggle Button Widget. . . . . . . . . . . . . . . . . . . 12

Figure 11. Toggle Buttons in a One-of-many Radio Box. . . . . . . . . . . 13

Figure 12. Toggle Buttons in a N-of-many Check Box. . . . . . . . . . . . . 13

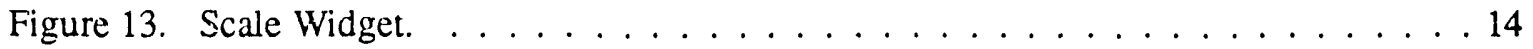

Figure 14. Scroll Bar Widget. . . . . . . . . . . . . . . . . . . . . . . 14

Figure 15. Scrolled List Widget. . . . . . . . . . . . . . . . . . . . . 14

Figure 16. Text Field Widget. . . . . . . . . . . . . . . . . . . . . . . 14

Figure 17. Scrolled Text Widget. . . . . . . . . . . . . . . . . . . 15

Figure 18. Popup Menu in an Application. . . . . . . . . . . . . . . . 15

Figure 19. Cascade Menu from the Menu Bar. . . . . . . . . . . . . . . . . . . . 16

Figure 20. Cascade Pullright Menu Before Pressing. . . . . . . . . . . . . . . 16

Figure 21. Cascade Pullright Menu After Pressing. . . . . . . . . . . . . . . . 16

Figure 22. Option Menu. . . . . . . . . . . . . . . . . . . . . 16

Figure 23. Option Menu After Popup. . . . . . . . . . . . . . . . . 17

Figure 24. An Application Using a Main Window. . . . . . . . . . . . . . . . . 17

Figure 25. Icons in a Scrolled Window Widget. . . . . . . . . . . . . . . 18

Figure 26. Motif Editor Using a Paned Widget. . . . . . . . . . . . . . . . . . 18

Figure 27. Selection Box Widget. . . . . . . . . . . . . . . . . . 19

Figure 28. File Selection Dialog. . . . . . . . . . . . . . . . . . . . 20

Figure 29. Command Widget. . . . . . . . . . . . . . . . . . . . . 20

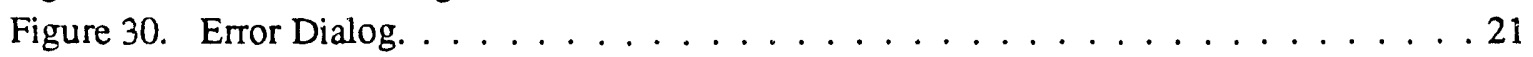

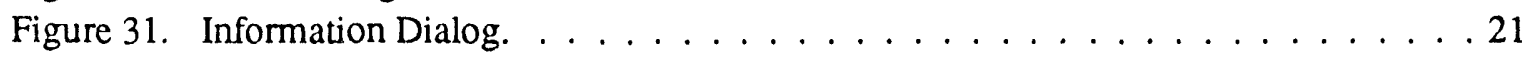

Figure 32. Question Dialog. . . . . . . . . . . . . . . . . . 22

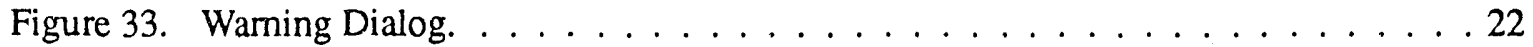

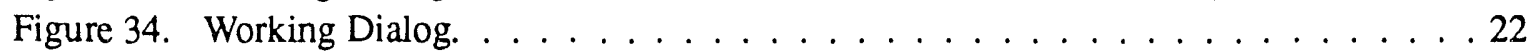




\section{Motif, the Basics \\ An Overview of the Widget Set}

\section{INTRODUCTION}

This report discusses using Motif for building a graphical user interface (GUI) for user applications. It contains information I leamed while using Motif for building an interface for several applications. After defining some terms, the following topics are addressed: a discussion of programming methodology, a comparison of Motif to other widget sets, Motif advantages and disadvantages, and finally, a listing of the major tools that programmers have at their disposal when putting together an interface. 


\section{WHAT IS MOTIF?}

The Motif library provides programmers with a rich set of tools for building a graphical user interface with a three-dimensional appearance and a consistent method for interaction for controlling an Unix application. Motif is an Xt-based, high-level library that gives programmers an "objectoriented" approach to program interface design (see Figure 1). Motif also allows end-users the flexibility to modify attributes (i.e., color, font, and text) of the interface.

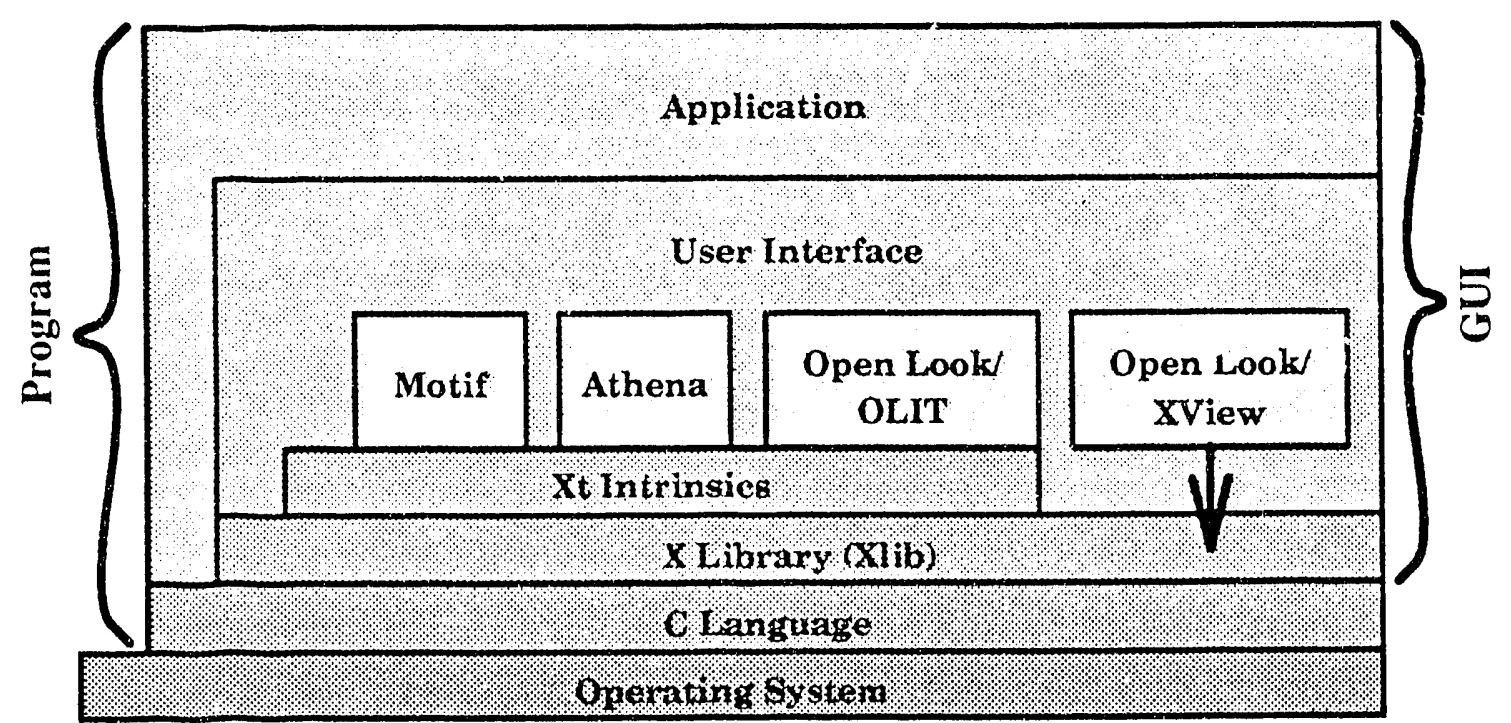

Figure 1. User Interface Model. 


\section{DEFINITION OF TERMS}

Like every technical field, the Motif and $\mathrm{X}$ world has its own unique jargon. The following terms are used throughout this document.

GUI - (pronounced GOOey) acronym for graphical user interface. GUIs differentiate themselves with bitmapped graphics that look like controls with which the user can interact (click a button, drag a scroll bar, etc.).

OSF - acronym for the Open Software Foundation, the organization that developed Motif.

Widget - a self contained "object". Widgets are basically the things in an application that a user interacts with. Push buttons, scroll bars, and toggle buttons, are widgets.

Widget Resources - attributes of the widget (e.g., color, font, and size) that can be set or modified by the user and/or programmer.

Compound Widget - a widget comprised of other widgets. The file selection dialog is an example of a compound widget.

Widget Hierarchy - defines the structure of how widgets are organized in a widget tree (see Figure 2).

Widget Parent/Child - describes the relationship between widgets in a widget tree. n Figure 2, for example, the "filePulldown" widget is i le parent of three children: "inputPushButton", "printPushButton", and "exitPushButton".

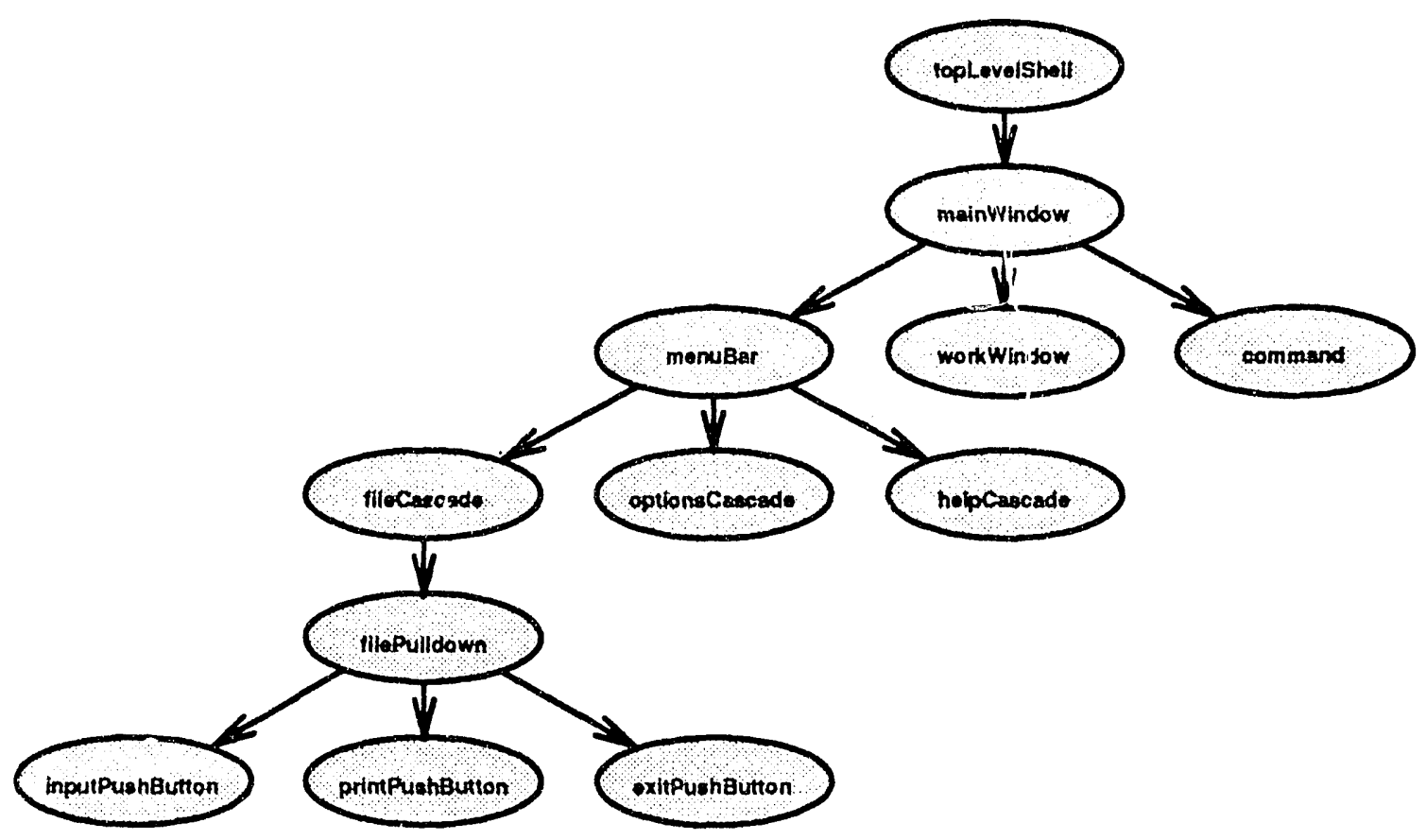

Figure 2. Widget Tree Hierarchy.

Look and Feel - refers to how the application appears (the look) and how the user interacts with the application (the feel). 


\section{PROGRAMMING METHODOLOGIES}

There are two main programming methodologies in existence: sequential and event-driven. Even though either aprroach may accomplish the same final results, there are obvious advantages of one methodology over another. The discussion that follows compares the two methods.

\subsection{Sequential Programming}

Standard (historical) character-based programs that display text to a terminal use the sequential methodology. Figure 3 illustrates standard sequential programming. A program that prescribes to this methodology prompts the user with a question, the user responds with an answer (usually terminated with a carriage return), and on it goes. The sequential nature of this environment puts the program in control and leads the user by the hand down a predetermined path. ${ }^{1}$

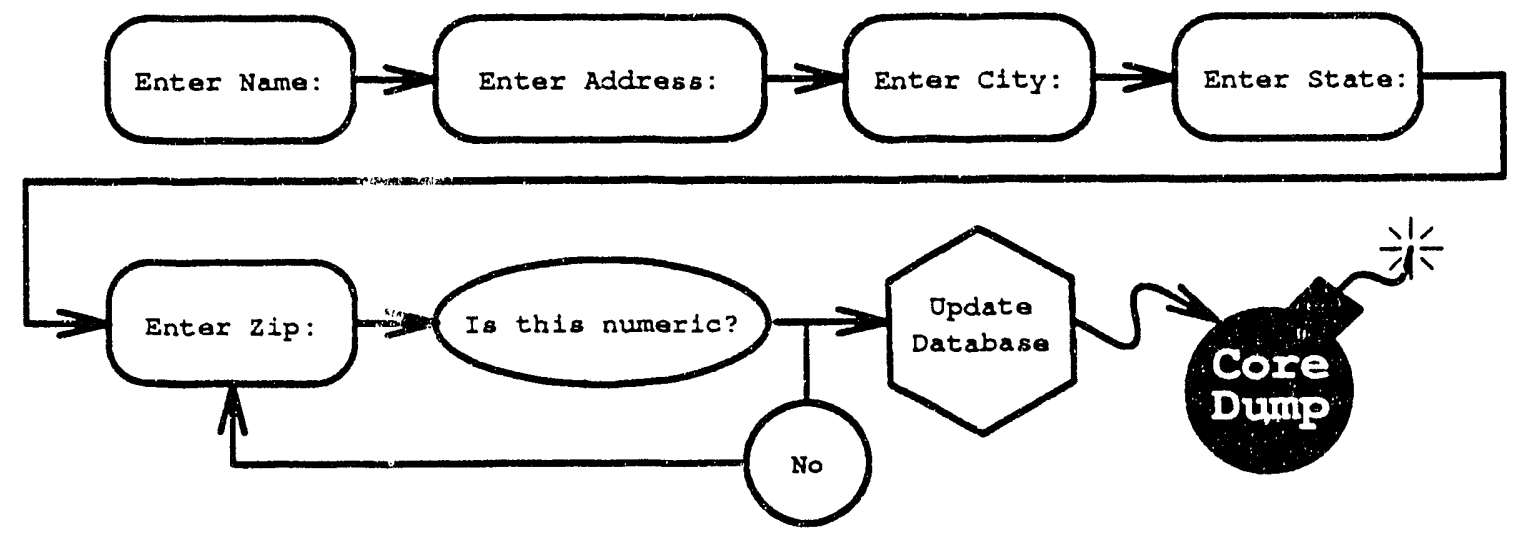

Figure 3. Standard Sequential Programming.

\subsection{Event-driven Programming}

The programming methodology that is used for a developing a graphical user interface is radically different from the programming methodology most of us learned in school. GUIs use an eventdriven methodology. Figure 4 illustrates event-driven programming. In this environment, the program functions like a switchboard that waits for user interaction (e.g., clicking a button or typing text). The user can enter the information in any order or not at all. Event-driven programs are much more user-centered; the program becomes the slave, not the user.

1. For an example of computer-centered programming, see also HAL in the movie "2001: A Space Odyssey". 


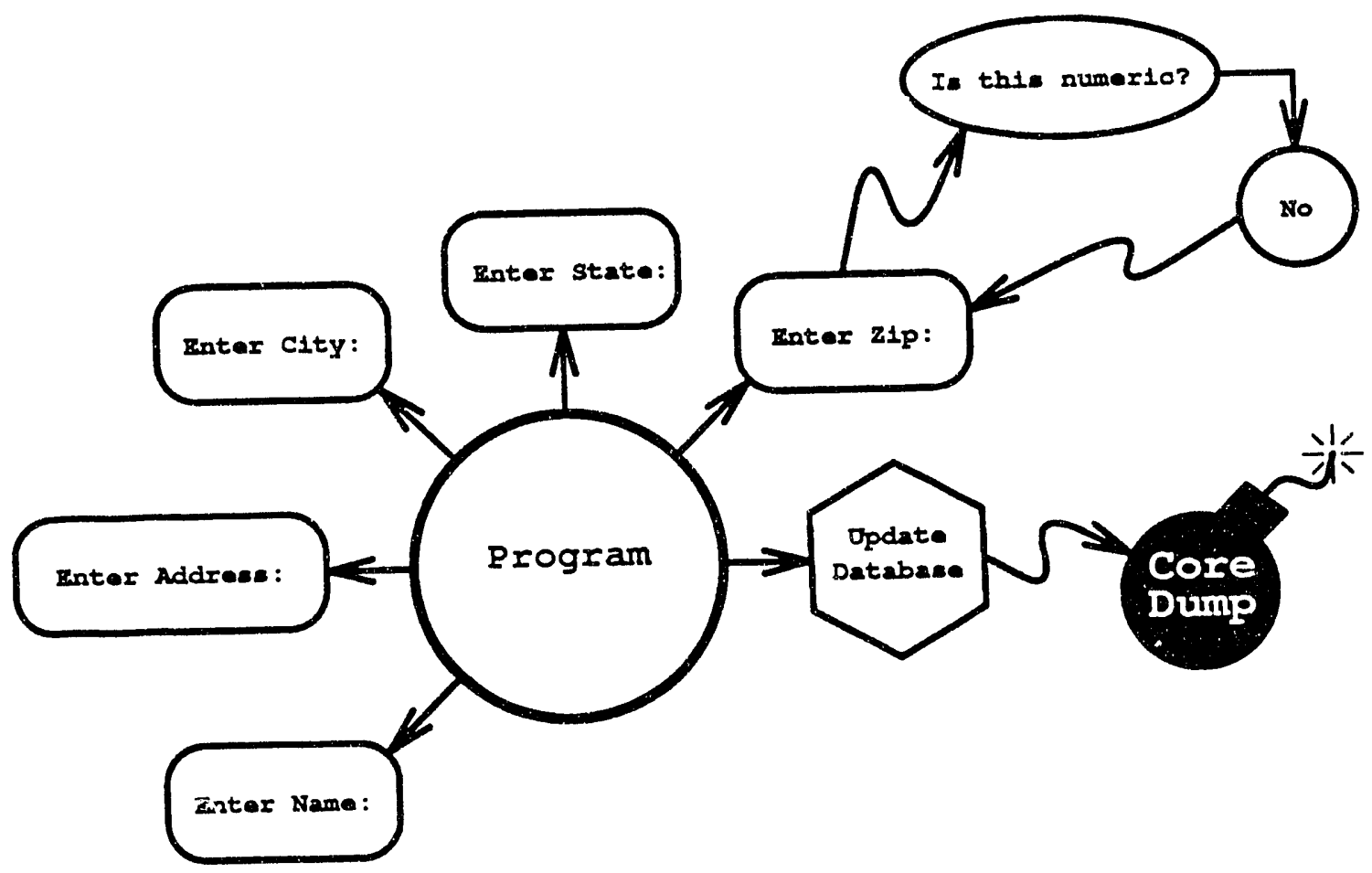

Figure 4. Event-driven Programming. 


\section{COMPARING THE WIDGET SETS}

For several years a war has been waging between commercial widget vendors regarding which product is superior. In the paragraphs that follow, I cut through all of the inarketing hype and offer some suggestions on comparing widget sets from an unbiased perspective.

\subsection{Evaluating the Look and Feel}

Most of the "look" and some of the "feel" issues are somewhat subjective. However, there are several noteworthy issues that should make the evaluation process more objective.

\subsection{1 "The Look" Considerations}

When evaluating the "look" (or the appearance! of a widget set, consider the following:

- Do all widgets have a shadow that makiz $u$ wr appear three-dimensional? The lack of a three-dimensional appearance to the widge $s$ makes an interface more difficult for some to use. When users encounter flat widgets, they must ask themselves, "Is this a button that I can interact with, or is this just a label that is presenting static information?"

- Do widget buttons have rounded corners? Are rounded comers important? Are rounded comers critical to my application? Can I live without them?

- How well do the widgets simulate similar controls from the physical world? If the application is a recorder of some sort, does the computer interface look like the buttons on a physical recorder? Users will go through less of a leaming curve and will find the application more intuitive if the user interface is similar to the control panel from the tangible world.

- When a button is activated, is there visual feedback? Can I tell if and when the button has been pressed?

- Does the visual appearance give accurate feedback regarding the state and function of the widget? Is the switch set or not set? What is the default/current selection?

Motif excels in all of these areas except for one. Motif widgets do not have a rounded shape.

\subsubsection{The "Feel" Considerations}

When evaluating the "feel" (or behavior) of a widget set, consider the following:

- What happens when I press a carriage return in this context? Can I predict with a good deal of certainty, before the fact, what is going to happen when the carriage return is pressed?

- What happens when I double (or triple) click in this context? Is this action consistent with how other widgets respond during multiple clicks?

- What happens when I press a tab or use the arrow keys in this context? Does control jump to the next field as in IBM applications? Users entering information from the keyboard may not want to move their hands to the mouse in order to enter information in the next field.

- Is the behavior consistent between different widgets throughout the application? For example, does cut and paste function the same way in all widgets? Does cut and paste work between the widgets? Between different applications? 
- Do widgets respond in a manner that is obvious/reasonable? Is the application easy to "drive"? Can I operate the buttons without a manual? Is it obvious how the widgets function? Is user interaction reasonable?

- Are keyboard accelerators available as short-cuts for the advanced user? A user that is familiar with an application may not need (or want) to sift through 3 levels of nested menus. Is there a way to execute a function directly without going through the menus?

- Can the user operate the interface completely without a mouse?

- Is the style of the application similar to interfaces in other environments? Would it be easy for a user to make the transition from a different hardware platform? Is this interface enough like other applications that the start-up will be minimal?

As you have probably guessed, Motif again meets all of thesc recommendations.

\subsection{Which is Better?}

It is just as difficult to answer the question, "Is Motif better?" as it is to answer, "Is chocolate better than vanilla?" The answer is highly dependent upon personal preference and greatly influenced by irrational prejudice not grounded in technical information (i.e., only boring people eat vanilla).

\subsection{Is it Easy to Write Motif Code?}

Because I have not written code using an Unix interface library other than Motif, I cannot honestly answer which interface library is easier to program. The Xt-based Open Look (OLIT) code and Motif code, however, do have similar calls, about the same number of widgets, and possess similar functionality. From a programmer's viewpoint, there is not a whole lot of difference between the two.

Some people have the preconceived notion that writing Motif code is very difficult. While writing Motif code is not trivial, for the most part it is easier to build a GUI in Motif than pure C, Xlib, or $\mathrm{Xt}$.

The following is an example source code listing of the simple Motif program pictured in Figure 5:

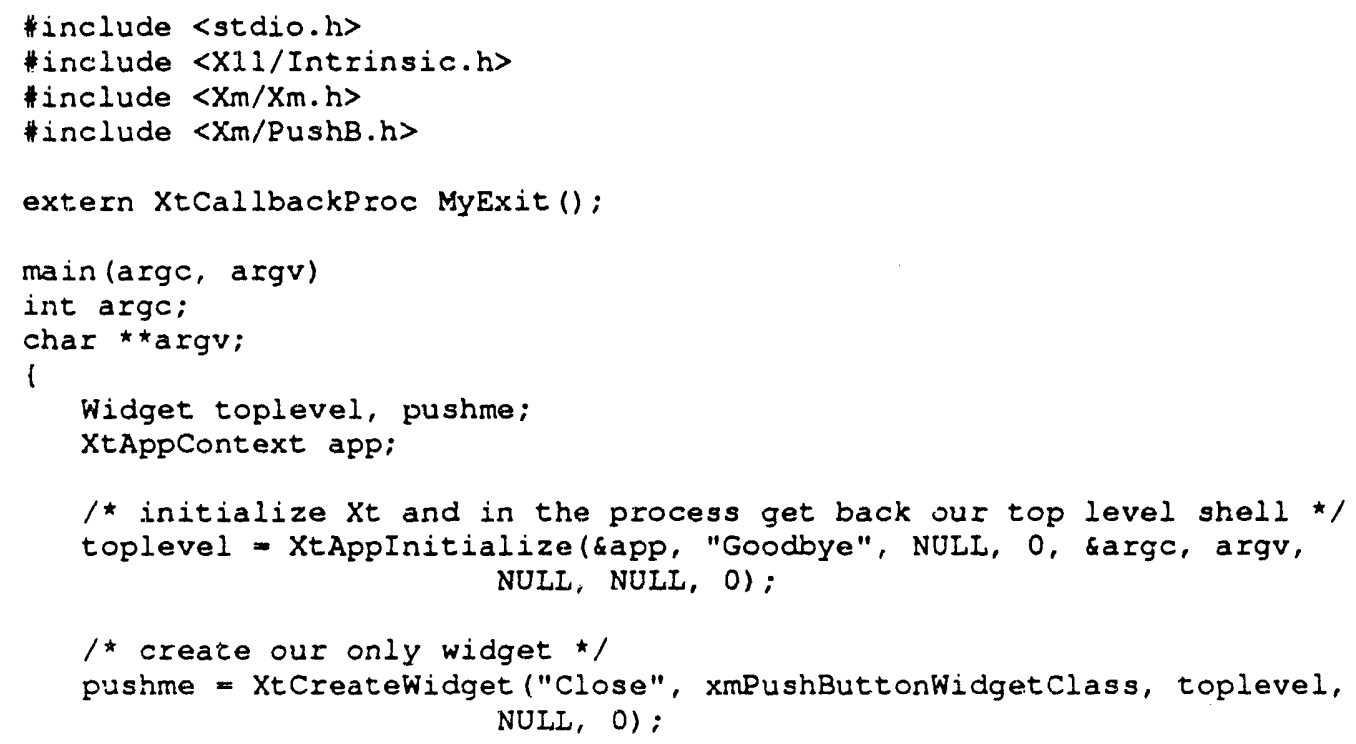




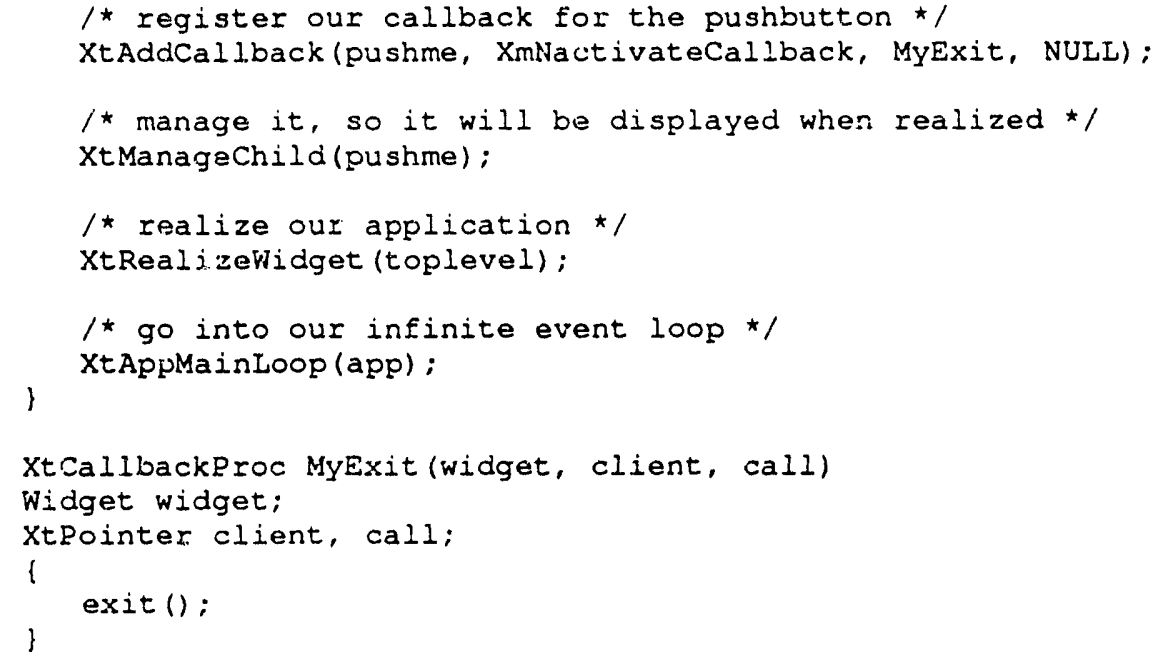

In the above application, one of the first things done is to include the Motif header files $\mathrm{Xm} . \mathrm{h}$ for the standard Motif definitions and PushB.h for the push button widget definitions. The specific header file for each widget class used must be included.

Next, notice that two widgets, toplevel and pushme are declared. The Xt call to XtAppInitialize creates toplevel the shell that is responsible for interfacing the window manager with the application. The only child of toplevel is pushme a push button widget. The parent of pushme is defined when the widget is created using $x t C r e a t e w i d g e t$. This widget could aiso be created by using the Motif convenience routine XmCreatePushButton.

Then the function call to MyExit is assigned to the button using $x t$ AddCallback. This informs the application to execute the user-defined function when the push button is pressed (or activated). The function MyExit, defined at the end of the program, performs all the work for the program.

The XtAppMainLoop tells the program to go into a "repeat forever" loop. The program then constantly waits for the user to perform an action.

This program creates a push button that terminates the entire application (exits) when it is pushed. I have been told that it would take about 90 lines of Xlib code to generate the same application. Motif encapsulates much xlib and Xt for you. This source code generates the application pictured in Figure 5.

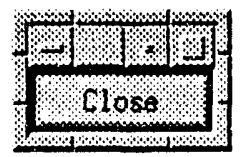

Figure 5. Simple Example Motif Application.

You should be able to compile this program using a small makefile or the following one line of code:

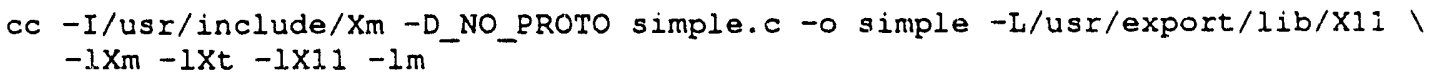




\section{WHAT ARE THE ADVANTAGES OF MOTIF?}

"If there is not a lot of difference between the Xt-based widget sets, why should I use Motif?" Consider the following points:

Abstraction: Motif's object-oriented approach gives programmers a great amount of high-level abstraction. If Motif programmers, for example, want to prompt the user for an existing filename, they simply pop up a file selection dialog. Programmers do not have to concern themselves with creating a widget that traverses a directory hierarchy. Programmers also do not have to create a filter that narrows down a list of filenames with the use of a wildcard. They merely create the widget with one call.

Appearance: The three-dimensional widget appearance is an attempt to simulate the appearance and behavior of physical control panel mechanisms for use in the computing environment. The three-dimensional buttons also assist the user in differentiating between widgets that require mouse interaction and the widgets that exist purely for informational purposes.

Availability: Several vendors are bundling the Motif libraries with their operating system. When porting an application, it would be undesirable for a developer to use a proprietary library that may not ve available on each machine in the environment.

Consistent Style: The OSF/Motif Style Guide and many of the widgets themselves encourage the conformance of a consistent look and feel (appearance and behavior) to the interface.

Flexibility: Programmers can allow the user to customize much of the application without recompiling. A color-blind user that cannot see a green background with a pink foreground, for example, can change it to something that is in their range of vision. A individual that is vision impaired may change the size and the style of the font to something that is more readable. A certain color may not be appropriate for some environments. A red waming dialog, for example, may be used to inform the user to prepare for a nuclear meltdown within the next 10 seconds. Instead of having all the dialogs appear in red, the discerning user may want to reserve the red dialog background color for severe wamings in order to distinguish it from other less important error messages.

Functionality: Motif has a large number of primitive and compound widgets to choose from. Many convenience routines (functions and macros) are also included that combine several standard $\mathrm{Xm} / \mathrm{Xt}$ calls into one (i.e., XmCreatesimpleoptionMenu, XmListDeleteAlIItems, XmselectionBoxGetChild, XmTextFieldGetString, etc.).

Interoperability: A Motif application will function in a near identical fashion regardless of which $\mathrm{X} 11$ window manager is running.

Open Solution: The libraries are available from several different vendors. This prevents programmers and users from getting locked into a proprietary library that is sold by a single vendor. The Motif source code is also widely available.

Portability: The Motif libraries have been ported to a large variety of Unix workstation architectures. Using the same Motif source code, the application should appear and function in same manner regardless of the platform. 


\section{WHAT ARE THE DISADVANTAGES OF MOTIF?}

By this time in the paper, you should be asking, "If Motif has so much going for it, why should I use anything else?" As hard as it may seem, there are a few draw backs with using Motif. Consider the following points:

Cost: If you are running on a hardware platform that does not have the Motif libraries bundled with the operating system (i.e., Suns) you must purchase the libraries from OSF or another vendor. This may be a disadvantage to those that are looking for a public domain solution (like the Athena widget set) off the network. The Motif libraries however, are very reasonable. A source license costs around $\$ 2,000$.

Size of Executable: The program executables tend to be very large. The simple example program pictured in Figure 5, was 434,000 bytes in size when compiled on a SPARCstation 1+. The size of that executable may have been reduced somewhat if unnecessary header files where not included and the extraneous libraries (like the math library) were not linked in.

Program Start-up: The start up time on a Motif program is going to be much greater than most sequential text-based programs. Motif programs usually require more memory and additional cpu cycles to create and manage the widgets. The simple example program pictured in Figure 5, took 2 to 3 seconds to display to the screen after it was executed on a SPARCstation 1+. Hopefully, the ease-of-use of the application will more than compensate for the inconvenience of the additional start up time. 


\section{MOTIF'S BAG OF TRICKS}

Mechanics on their first day of work in a large commercial shop may find themselves using only a limited number of the tools only because they do not know what is available. In addition, inexperienced mechanics may use a wrench for hammering not because it is the tool that is the best suited, but because it is convenient. Motif programmers r: zy experience the same pitfalls.

One key to Motif programming proficiency is to know the gamut of tools that are available. Just as a seasoned mechanic uses the proper tool for the occasion, it is just as important to use the right widget for the right job. Many make-shift tools that programmers inadvertently construct have already been written and are available as standard widgets. Major gains in productivity can also be achieved when programmers use built-in convenience routines instead of repeatedly "hacking" something together.

I have divided the widgets into four different categories: primitive widgets, menu widgets, manager widgets, and dialog widgets. The categories are somewhat arbitrary and there is a good deal of overlap between the divisions. The following sections provide some of the most often used Motif widgets in those categories.

\subsection{Primitive Widgets}

Primitive widgets are the "least common denominator" widgets that become the basic building blocks that are used to fabricate an application.

The primitives addressed in section 8.1.1 through section 8.1.5 are push button-like widgets that possess many similar characteristics. A color pixmap can be used instead of a text label on all the following push buttons. The push button-like widgets are the label, push button, drawn button, arrow button, and toggle button. Additional primitive widgets are mentioned in section 8.1.6 through section 8.1.9.

\subsubsection{Label}

The label widget is a static (non-interactive) widget that is used for portraying information to the user regarding specific details of the application (see Figure 6). Similar to a label on a consumer product, the label widget tell users what the application is, what it does, how it operates, etc.

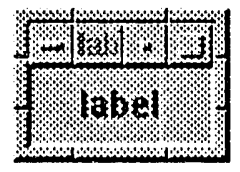

Figure 6. Label Widget.

\subsubsection{Push Button}

The push button widget is probably the most often used interactive widget in an application (see Figure 7). It comes as standard equipment on many of the compound widgets. Push buttons are also used on cascade, popup, and option menus. 
Figure 7. Push Button Widget.

\subsubsection{Drawn Button}

A drawn button widget looks similar to the push button widget, but it has more options for the button shadow (see Figure 8). The drawn button also has callbacks to support drawing operations.

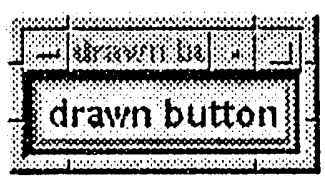

Figure 8. Drawn Button Widget.

\subsubsection{Arrow Button}

The arrow button widget is essentially a push button widget equipped with an arrow pixmap (see Figure 9).

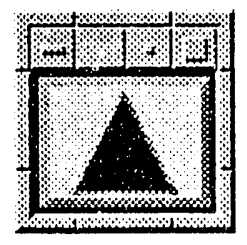

Figure 9. Arrow Button Widget.

\subsubsection{Toggle Button}

A toggle button widget is a binary switch that records a state change (see Figure 10). Toggle buttons can also be combined to function together in two different manners:, as a radio box or check box.

Figure 10. Toggle Button Widget.

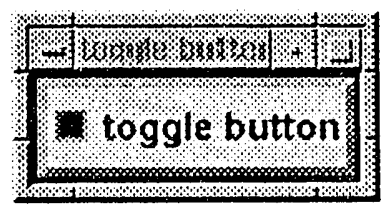

\subsubsection{Radio Boxes.}

Radio boxes are a series of toggle buttons that operate like old-fashion car radio switches that changed stations. Only one button can be selected at a time. When a new button is selected, the previously selected button is pushed out or unselected (see Figure 11). The state buttons in a radio box are a diamond shape. 


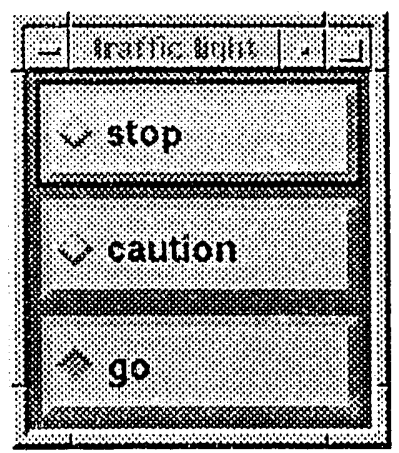

Figure 11. Toggle Buttons in a One-of-many Radio Box.

\subsubsection{Check Boxes.}

Check boxes are a series of toggle buttons that function independently of each other. In other words, multiple buttons can be selected (and unselected) (see Figure 12). The state buttons in a check box are a rectangular shape.

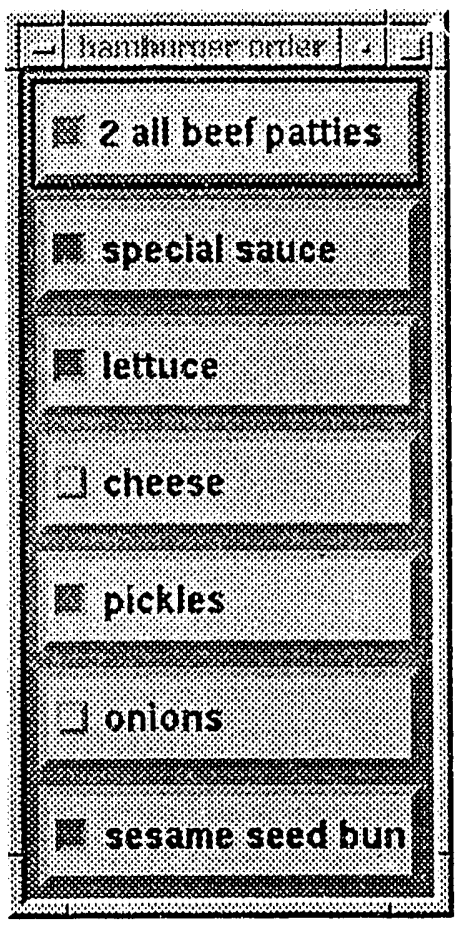

Figure 12. Toggle Buttons in a N-of-many Check Box.

\subsubsection{Scale}

A scale widget is useful for setting a value in a finite range (see Figure 13). The value on the scale is updated as the user drags the slider with the mouse. When the sensitivity is tumed off, the scale can also be used as a display-only gauge to indicate the current value. 


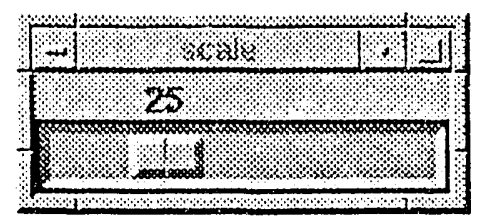

Figure 13. Scale Widget.

\subsubsection{Scroll Bar}

The scroll bar is a primitive widget that is also used as a component in many compound widgets. It is used for building a scrolled context in an application (see Figure 14).

Figure 14. Scroll Bar Widget.

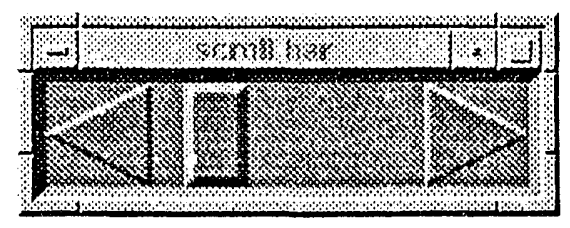

\subsubsection{List Widget}

The list widget is useful for permitting the user to select an item (or items) off a list (see Figure 15).

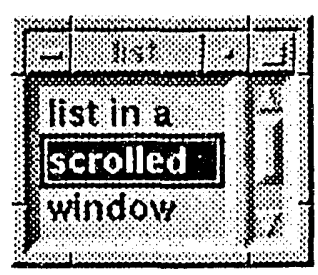

Figure 15. Scrolled List Widget.

\subsubsection{Text Widgets}

A text widget is used to accept keyboard entry from a user. The Motif text widget incorporates some very powerful editing capabilities, many of which can be customized by the user. The two most commonly used text widgets are the text field and the scrolled text.

\subsubsection{Text Field.}

The text field widget is used when small amounts of text (no more than one line) are requested by the program (see Figure 16).

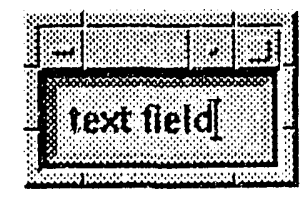

Figure 16. Text Field Widget. 


\subsubsection{Scrolled Text.}

The scrolled text widget is used when multiple lines of text are requested by the program (see Figure i7).

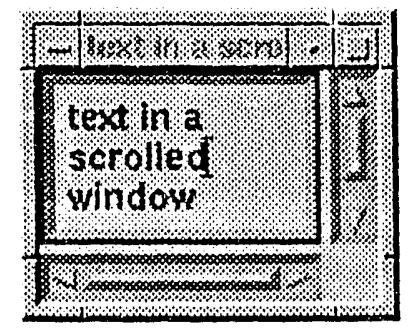

Figure 17. Scrolled Text Widget.

\subsection{Menu Widgets}

Similar to selecting items off a menu at your favorite greasy spoon, you can also select items off a Motif menu. The common menus available are the popup menu, the cascade menu, and the option menu.

\subsubsection{Popup Menu}

A popup menu is handy for those applications where programmers do not want to reserve screen real estate for a menu. Users do not have to move their mouse; they activate (pop up) the menu by pressing the appropriate mouse button (mouse button three is usually reserved for this function). On an editor, for example, a popup menu is handy so that the menu does not permanently conceal the text file (see Figure 18).

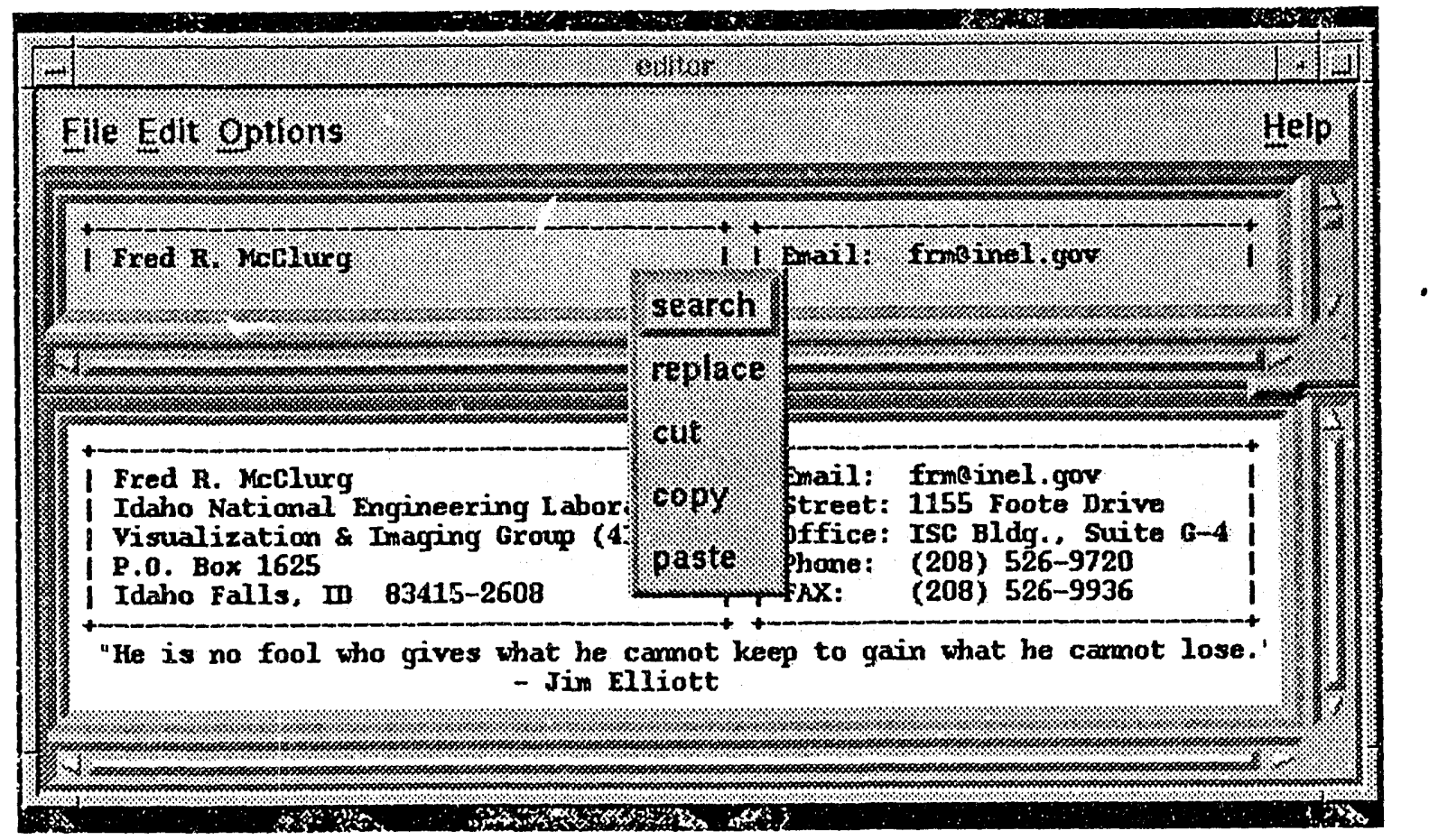

Figure 18. Popup Menu in an Application. 


\subsubsection{Cascade Menu}

Cascade (also known as pulldown) menus come down like a retractable shade on a window. Cascade menus most often originate on a menu bar located at the top of the application window (see Figure 19).

Figure 19. Cascade Menu from the Menu Bar.

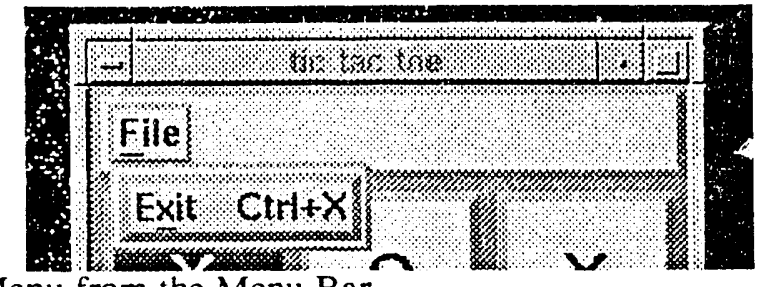

Cascade menus can also be nested. When one of the push buttons has a cascade menu as a descendant, it is known as a pullright menu. An arrow icon on the right side of the push button indicates the presence of a pullright menu (see Figure 20).

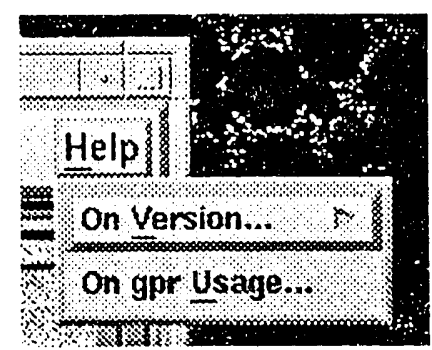

Figure 20. Cascade Pullright Menu Before Pressing.

Pressing a pullright menu reveals an attached popup menu (see Figure 21). Pullright menus are a handy way to pack a lot of information into a menu. It also gives programmers a method of organizing the menu into categories.

Figure 21. Cascade Pullright Menu After Pressing.

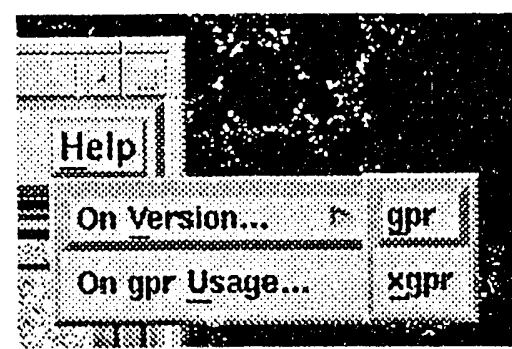

\subsubsection{Option Menu}

An option menu is a push button with a popup menu attached. This menu is differentiated from a push button widget by a sash on the right of the option menu (sec Figure 22).

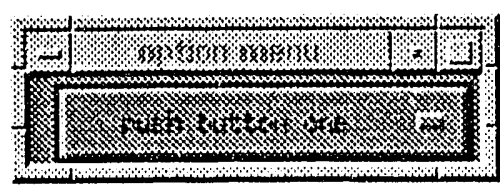

Figure 22. Option Menu. 
The popup menu is displayed when the option menu is pressed (see Figure 23). When a menu item is selected, that choice becomes the current label on the optior menu. This history mechanism gives the user a visual cue regarding the current state or the previous menu selection.

The option menu can function as a one-of-many radio box without taking up as much screen real estate.

Figure 23. Option Menu After Popup.

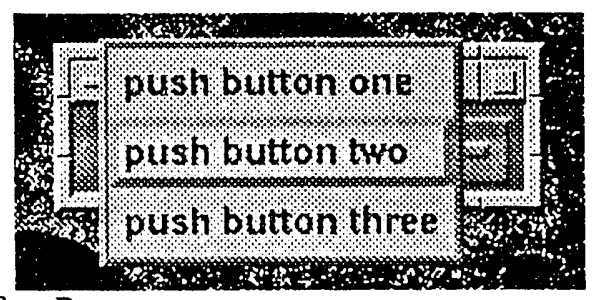

\subsection{Manager Widgets}

Manager widgets control and organize children. They act as containers to organize the widgets together. Some managers have no potential for interaction (i.e., no open-door policy). A bulletin board manager widget is used for "tacking up" widg.t children at random locations. A form manager widget controls the arrangement of widget children by establishing a relationship between them. A row column manager widget encourages the children to fall into line by organizing them in neat rows and columns.

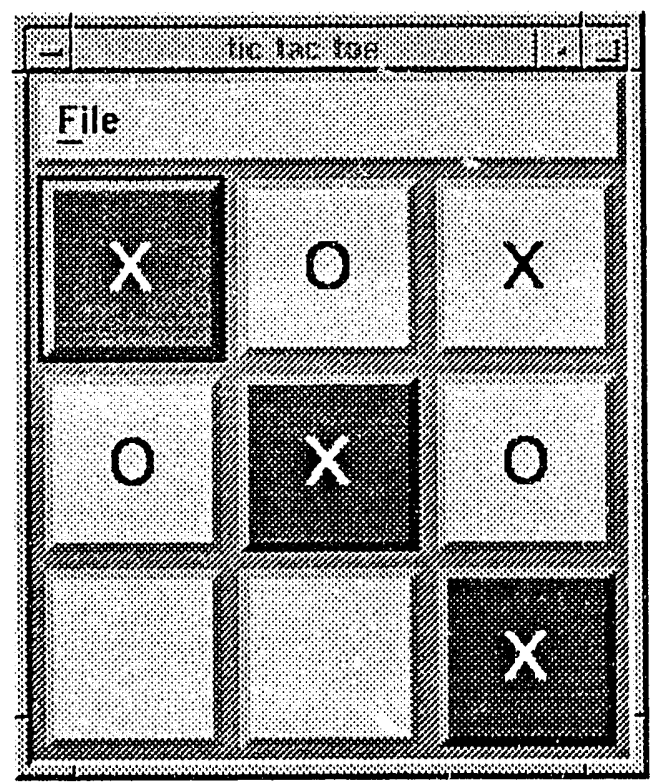

Figure 24. An Application Using a Main Window.

\subsubsection{Main Window}

The main window manager widget strongly encourages the programmer to conform to the Motif Style Guide for applications using a main window. This widget reserves the top portion of the window for the menu bar, the middle portion for a work area, and the lower portion for a command and message area. The work area of the main window pictured in Figure 24, organizes the push buttons by using a row colımn manager widget. 


\subsubsection{Scrolled Window}

A scrolled window manager widget is useful for giving the user a viewport into a larger window. The icon box from the Motif window manager is a good example of application using a scrolled window widget (see Figure 25).

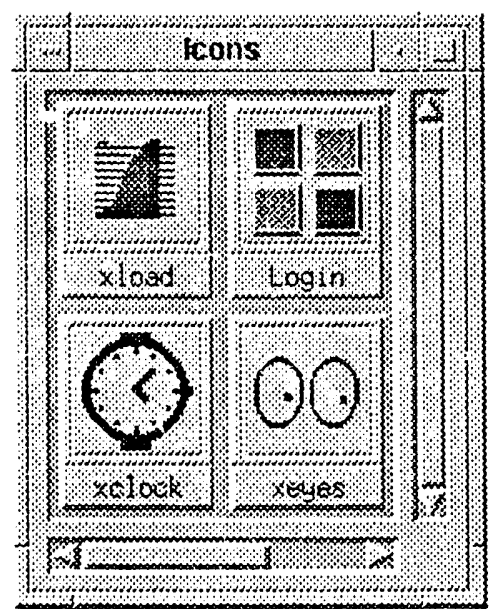

Figure 25. Icons in a Scrolled Window Widget.

\subsubsection{Paned Window}

A paned window is a widget that can possess only two children and has a sash that is used to adjust the size of the two children (see Figure 26). When the sash is moved, cne child widget gets smaller (becomes less visible) while the other child becomes larger (becomes more visible). A paned window is usually used to partition the window into two parts to provide a mechanism for sharing the same screen real estate.

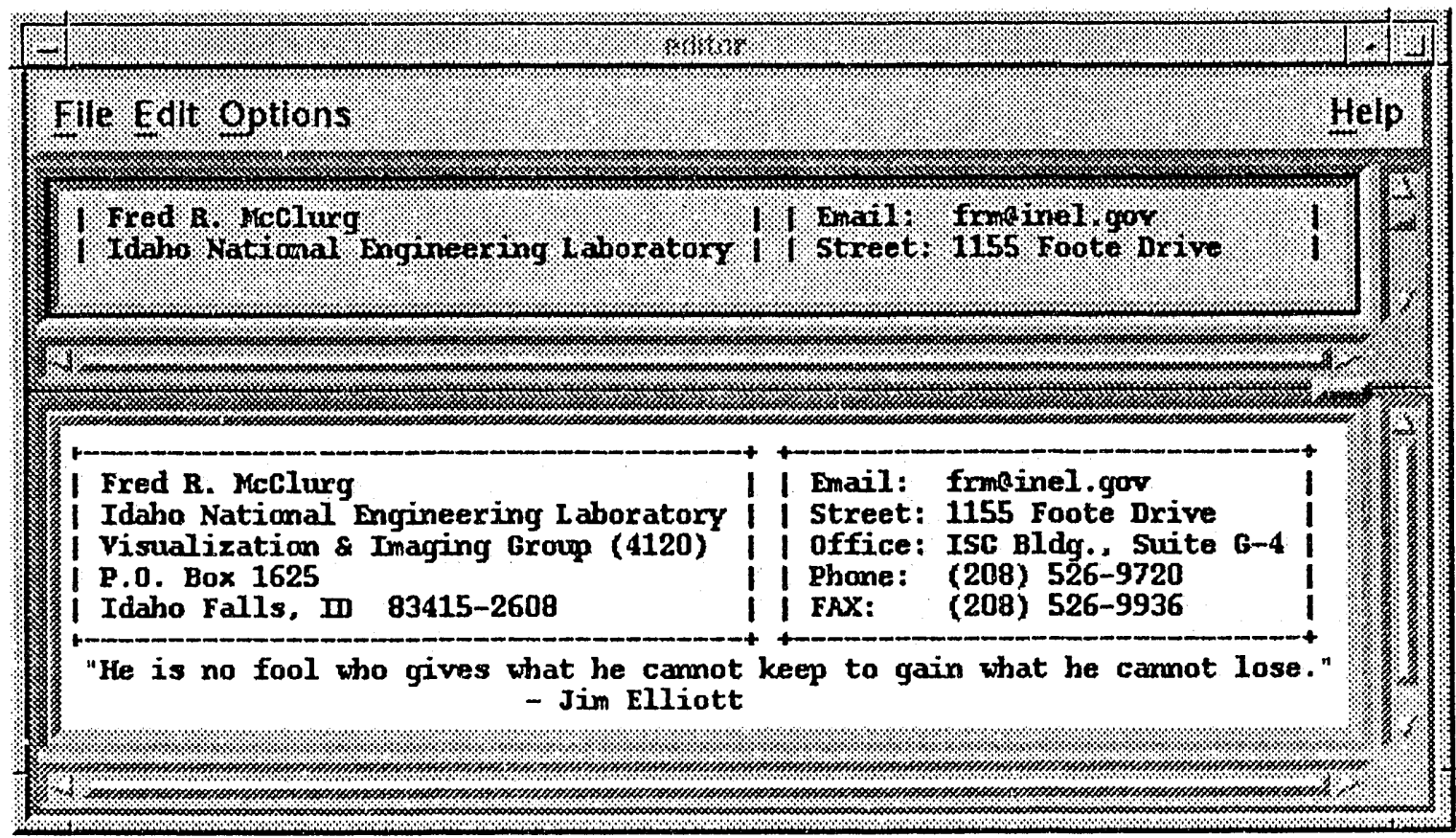

Figure 26. Motif Editor Using a Paned Widget. 


\subsection{Dialog Widgets}

Dialogs are compound widgets that encapsulate a great deal of high-level functionality by combining many primitive widgets. All of the dialogs can be customized to meet specific requirements. Motif provides these widgets as a convenience to the programmer.

\subsubsection{Selection Dialog}

The selection dialog is used for inputting new items, selecting items off a list, and/or editing those items (see Figure 27).

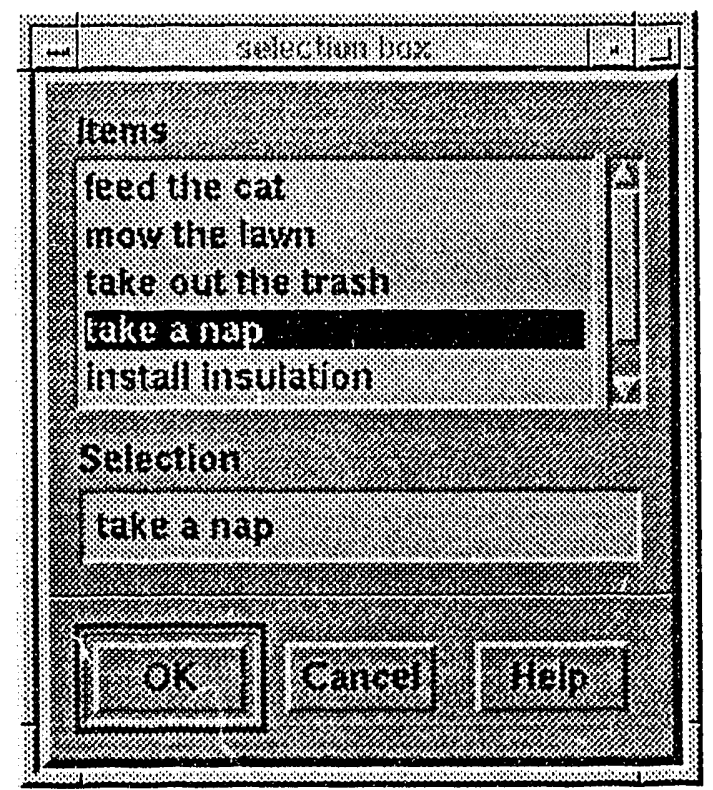

Figure 27. Selection Box Widget.

\subsubsection{File Selection Dialog}

Most applications require the user to input an existing filename. Motif has a convenience that does it for you. The file selection dialog is a handy little widget that allows the user to traverse a directory hierarchy and select an existing file (see Figure 28). This dialog also allows you to specify a wildcard as a filter to obtain a subset of the directory list and narrow down the selection process. 


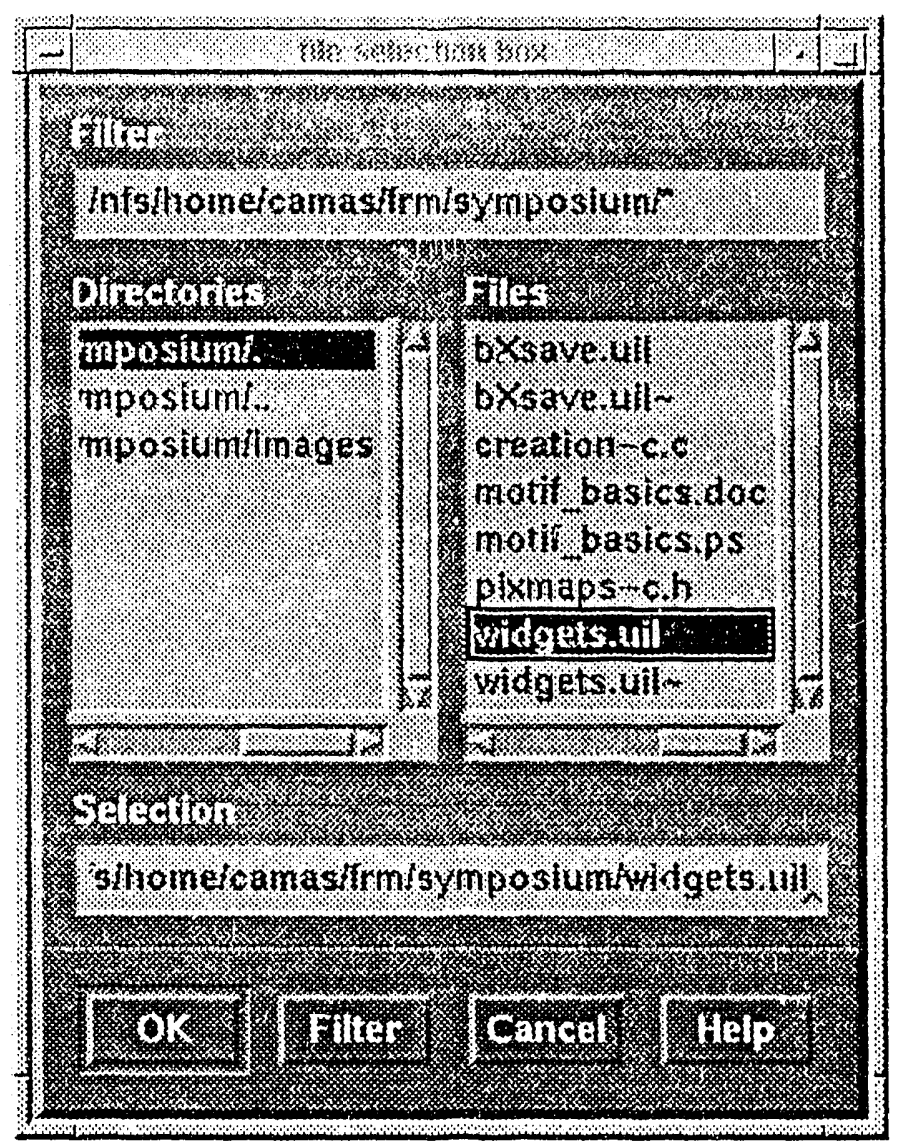

Figure 28. File Selertion Dialog.

\subsubsection{Command Dialog}

The command dialog is used to issue a command in an application (see Figure 29). This dialog has a built-in history mechanism that keeps track of previously entered commands. Previous commands can be selected from a list, edited, and re-executed.

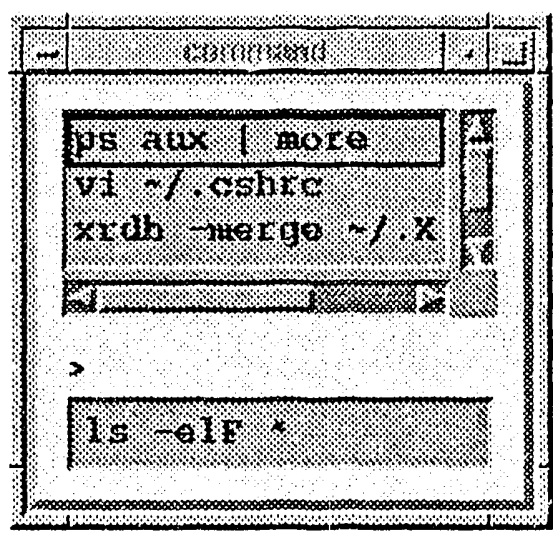

Figure 29. Command Widget. 


\subsubsection{Message Dialogs}

Message dialogs are popup windows that are used to instruct the user about some detail of operation regarding the program. The speciality dialogs listed below come with an icon (that can be changed) that give the user a feel for the message caregory.

Each of the following message dialogs can be modal or modeless. A modal dialog forces the user to reply to the dialog by not allowing interaction with any other part of the application. Most of a GUI application however, is expected to be modeless.

\subsubsection{Error Dialog.}

The error dialog is used for reporting error messages (see Figure 30).

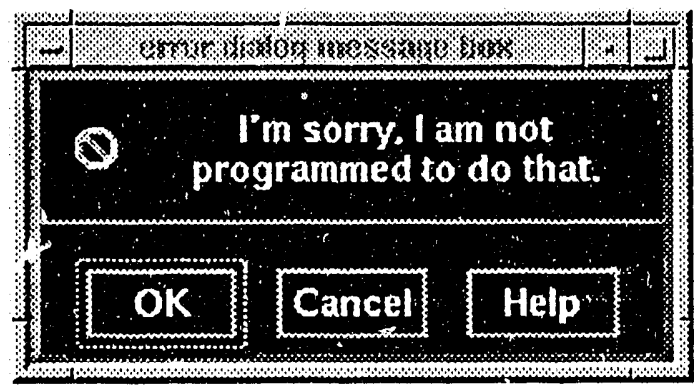

Figure 30. Error Dialog.

\subsubsection{Information Dialog.}

An information dialog is used to portray benign (but hopefully useful) information to the user (see Figure 31).

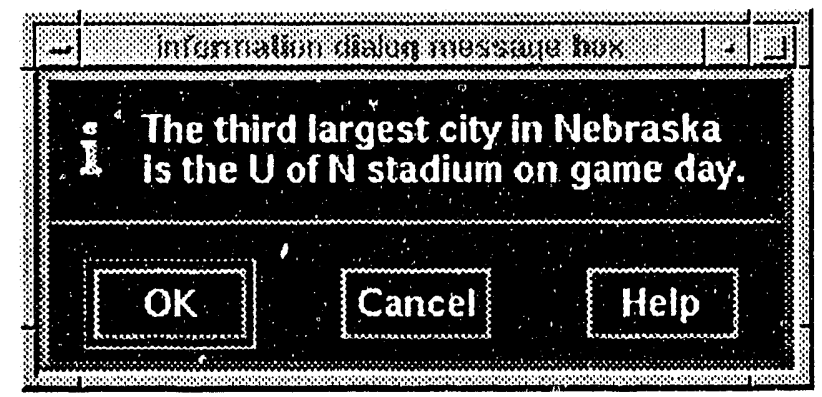

Figure 31. Information Dialog.

\subsubsection{Question Dlalog.}

The question dialog prompts the user with a question (see Figure 32). 


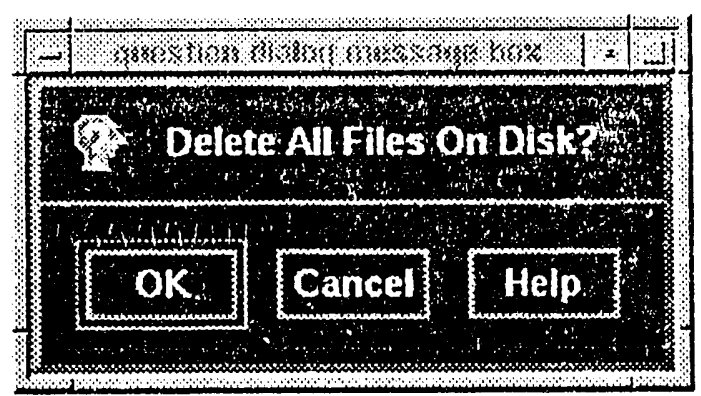

Figure 32. Question Dialog.

\subsubsection{Warning Dialog.}

The warning dialog should be used to shock the user regarding impending doom (see Figure 33).

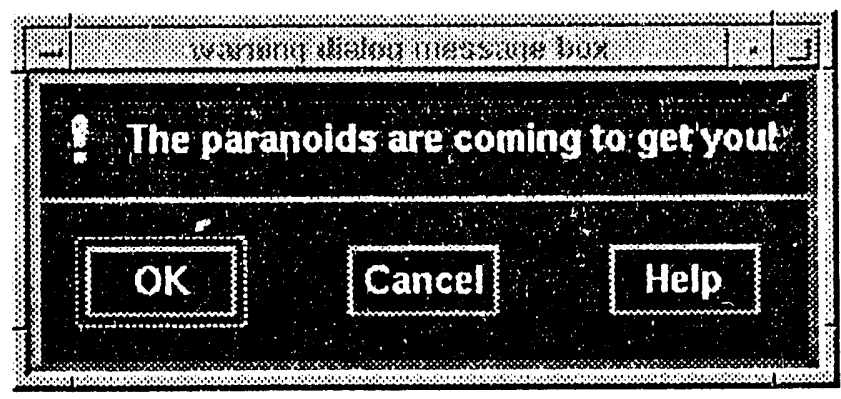

Figure 33. Waming Dialog.

\subsubsection{Working Dialog.}

The working dialog is used to present a polite "Please Wait" message to the user during computational intensive periods (see Figure 34).

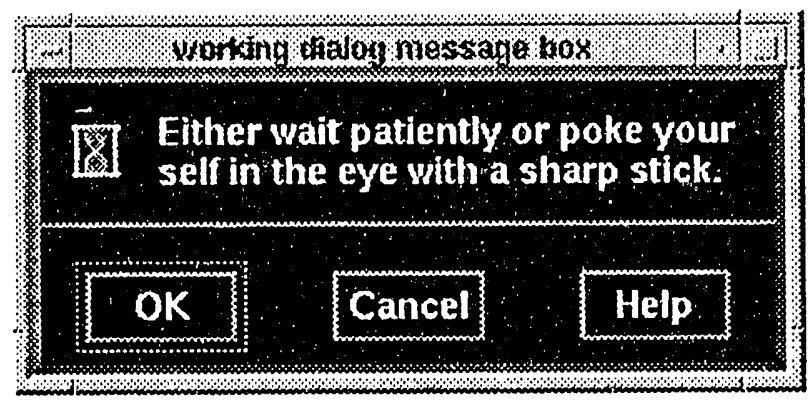

Figure 34. Working Dialog. 


\section{CONCLUSION}

I encourage you to consider using Motif for your next project. It is a very flexible, widely available, and extremely portable way to build a user interface. Rumors that Motif is riddled with bugs are greatly exaggerated. On the contrary, I found that the Motif widgets were very well thought out and extremely usable. Most of the problems (e.g., core dumps) that I encountered were related to my lack of experience in Xt and $\mathrm{C}$, not with Motif.

Motif can open up a brave new world for your applications. 


\section{BIBLIOGRAPHY}

Heller, D., 1991, Motif Programming Manual, Volume Six, Sebastopol, California: O’Reilly \& Associates, Inc.

Hopeman, C. and D. Shaffer, 1992, "Programming the Motif Toolkit Parts 1 \& 2", Xhibition '92, San Jose, California, June 15-19, 1982.

Johnson, E. and K. Reichard, 1991, Power Programming... MOTIF, Portland, Oregon: Management Information Source, Inc.

Kimball, P., 1992, "Programming X, Part 1: Introduction to the X Toolkit", Xhibition '92, San Jose, California, June15-19, 1982.

Open Software Foundation, Inc., 1990, OSF/Motif ${ }^{\mathrm{TM}}$ User's Guide, Revision 1.0, Englewood Cliffs, New Jersey: Prentice Hall Publishing Company, Inc.

Open Software Foundation, Inc., 1991, OSF/Motif ${ }^{\mathrm{TM}}$ Application Environment Specification (AES) User Environment Volumme, Revision B, Englewood Cliffs, New Jersey: Prentice Hall Publishing Company, Inc.

Open Software Foundation, Inc., 1991, OSF/Motif TM Programmer's Guide, Revision 1.1, Englewood Cliffs, New Jersey: Prentice Hall Publishing Company, Inc.

Open Software Foundation, Inc., 1991, OSF/Motif ${ }^{\mathrm{TM}}$ Programmer's Reference, Revision 1.1, Englewood Cliffs, New Jersey: Prentice Hall Publishing Company, Inc.

Open Software Foundation, Inc., 1991, OSF/Motif ${ }^{\mathrm{TM}}$ Style Guide, Revision 1.1, Englewood Cliffs, New Jersey: Prentice Hall Publishing Company, Inc.

Sobell, M., 1989, A Practical Guide to the UNIX System, 2nd ed., Redwood City, California: Benjamin/Cummings Publishing Company, Inc.

Talbott, S., 1988, Managing Projects with Make, Newton, Massachusetts: O'Reilly \& Associates, Inc. 

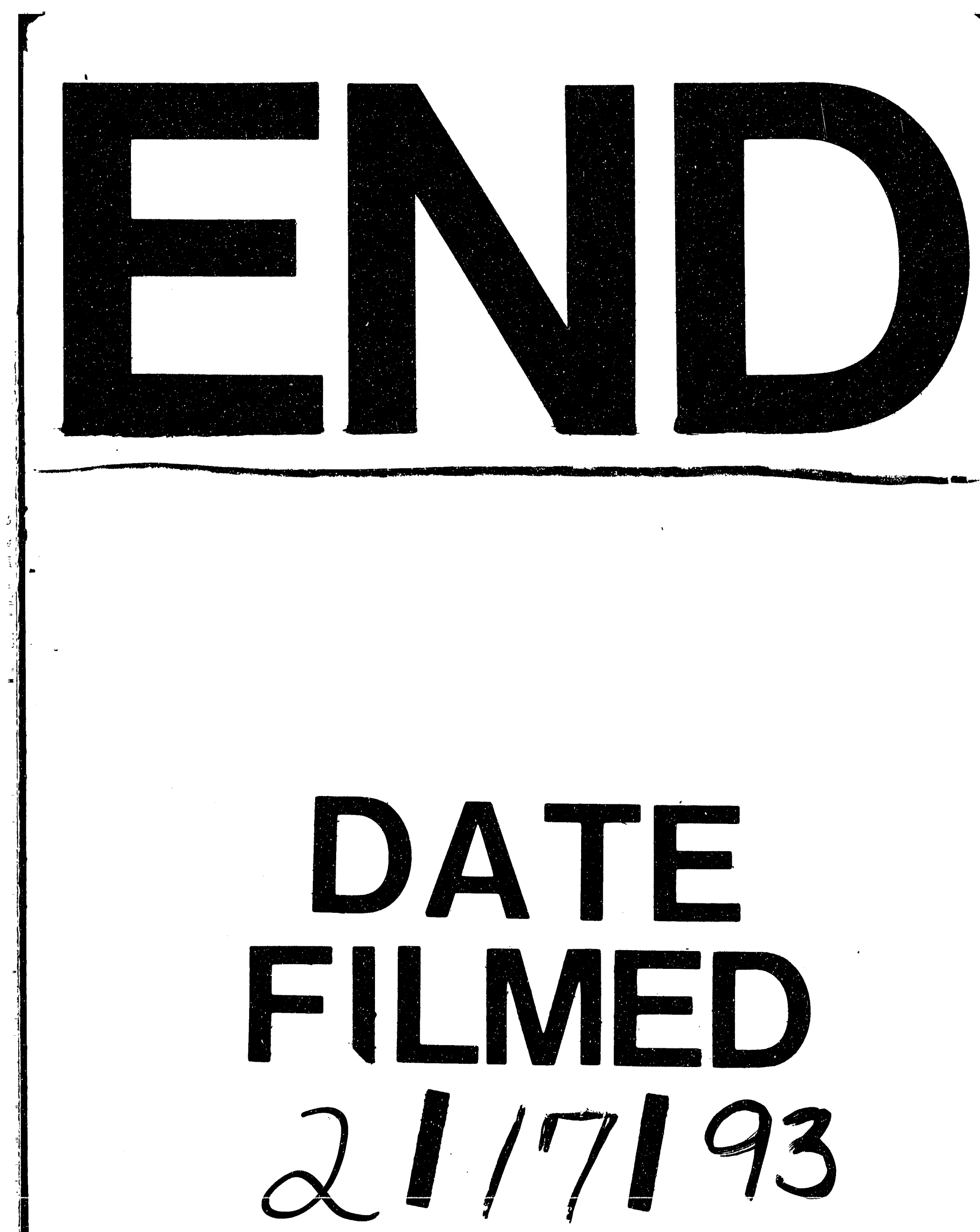


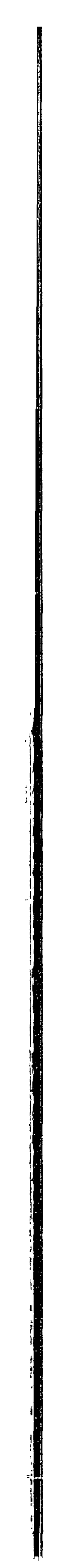

